\section{MS23-P3 Crystal orientation mapping on metallic nanoparticles by electron diffraction methods}

Arturo Ponce ${ }^{1}$, Muriel Veron², Francisco Ruiz-Zepeda ${ }^{1}$, John E. Sanchez ${ }^{1}$, Ulises Santiago ${ }^{1}$, Fernando Mendoza-Santoyo ${ }^{1}$, Jesus Velazquez-Salazar $^{1}$, Miguel Jose-Yacaman ${ }^{1}$

1. Department of Physics and Astronomy, University of Texas at San Antonio, One UTSA Circle, San Antonio, Texas 78249, USA.

2. Laboratoire SIMaP, Grenoble INP - CNRS - UJF, Saint Martin D'Hères Cedex, France

\section{email: arturo.ponce@utsa.edu}

Electron diffraction in transmission electron microscopy (TEM) provides crystallographic information of the specimens. Lately, new methodologies in electron diffraction such as diffraction in scanning modes, the use of aberration correctors to sharpen the probe size and precession electron diffraction (PED) among others have been becoming more frequent for the characterization of nanostructured materials. On the other hand, for data collection new detectors have been also developed with high sensitivity and high speed to collect intensities from the electron diffraction patterns in nanometric sized and sensitive materials like in metallic nanoparticles. In the present work we determine the geometry of modified gold decahedra nanoparticles by using crystalline orientation mapping and coherent electron diffraction. In both cases, nanoarea electron diffraction (NED) and small condenser aperture have been used, which reduce the diameter of the electron beam to a few nanometers. Coherent electron diffraction shows streaks lines, which have been measured and related to high index crystallographic planes in multiple faceted nanoparticles. The analysis of the geometry of these nanoparticles has been performed from the indexing of multiple electron diffraction patterns collected through PED and then raster the electron beam through the area of interest on the sample. The patterns are recorded in an external ultrafast CCD camera attached to the viewing screen of the microscope and every electron diffraction pattern is registered at each step of the scanned and stored in a computer for a subsequent indexing process. The result can then be displayed as a phase or an orientation map of the scanned area as can be observed in the color map of the Figure 1. The pattern recognition is done by calculating a cross correlation and a reliability value for each experimental pattern with crystallography open database. The particle has been oriented in the five-fold symmetry and lateral view. The maps obtained are analyzed and correlated with the projection of the different orientations of the crystals within the nanoparticle.

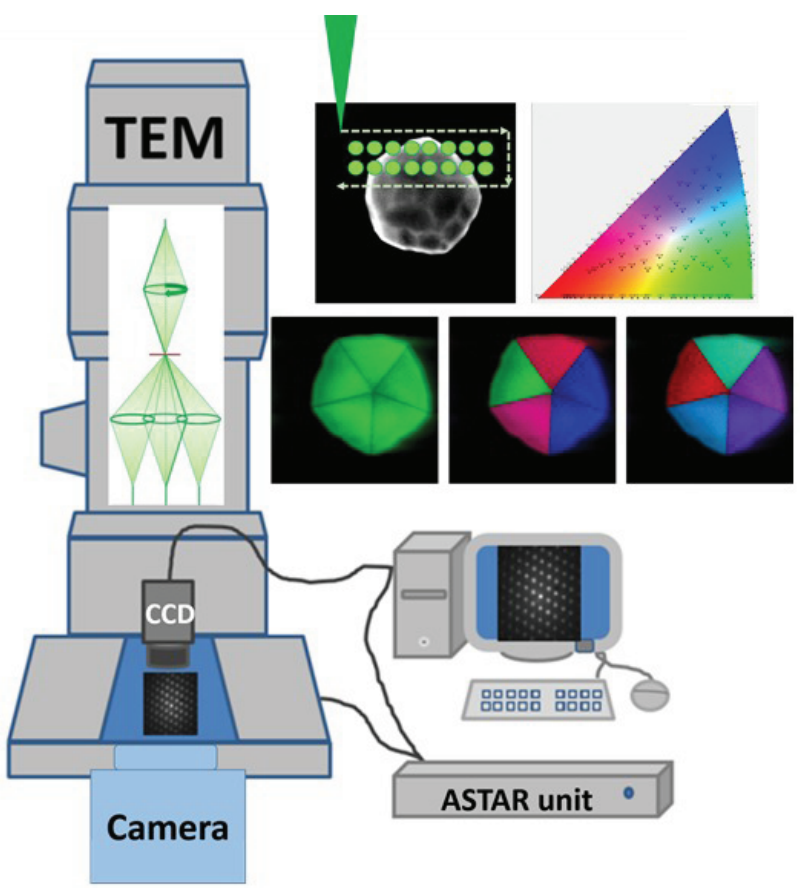

Figure 1. Schematic representation the ASTAR-precession electron diffraction system attached to a transmission electron microscope and the orientation maps of a decahedral gold nanoparticle oriented in the five-fold symmetry and its pole figure color chart.

Keywords: decahedral nanoparticles, electron diffraction, crystal orientation maps, precession electron diffraction 